\title{
Sonographic assessment of fetal head deflexion using occiput: spine angle measured during first stage of labour and its role in predicting the mode of delivery among nulliparous women
}

\author{
Keerthi Somu, Sujatha B. S.*, Shripad Hebbar, Shyamala G., Muralidhar V. Pai
}

Department of Obstetrics and Gynecology, Kasturba Medical College, Manipal, Manipal Academy of Higher Education, Manipal, India

Received: 26 June 2019

Accepted: 03 July 2019

*Correspondence:

Dr. Sujatha B. S.,

E-mail: bssujata@gmail.com

Copyright: () the author(s), publisher and licensee Medip Academy. This is an open-access article distributed under the terms of the Creative Commons Attribution Non-Commercial License, which permits unrestricted non-commercial use, distribution, and reproduction in any medium, provided the original work is properly cited.

\begin{abstract}
Background: The attitude of the fetal head during labour significantly influences the progress and outcome of delivery and is mainly diagnosed by vaginal examination during labour. The aim of the study was to quantify the extent of deflexion of the fetal head by measuring the fetal occiput spine angle (OSA) through transabdominal ultrasonography in the first stage of labour and to determine whether the fetal OSA can predict the mode of delivery. Methods: We conducted a prospective observational study on 145 nulliparous uncomplicated singleton pregnant women without occiput-posterior position of the fetus during active labour. The OSA was measured as the angle between the two tangential lines to the occipital bone and the vertebral body of the first cervical spine, during active labour and monitored until delivery. Intra- and interobserver reproducibility of the OSA measurement and the correlation between the OSA and mode of delivery were also evaluated.

Results: For the study population, the mean value of the OSA measured in the active phase of the first stage was $124.2 \pm 11.5^{\circ}$. The OSA measurement showed excellent intraobserver agreement $(\mathrm{r}=0.82 ; 95 \%$ confidence interval [95\% CI] 0.70-0.80) and fair-to-good interobserver agreement $(\mathrm{r}=0.62 ; 95 \% \mathrm{CI} 0.51-0.71)$. The mean OSA was significantly less for the group of patients who required conversion to cesarean section due to labour arrest $(n=32)$ as compared to those who had vaginal delivery ( $\mathrm{n}=113)\left(116.25 \pm 9.2^{0}\right.$ versus $\left.126.53 \pm 11.1^{0}, \mathrm{P}<0.01\right)$. An OSA of $\geq 121^{\circ}$ was associated with vaginal delivery in $80.5 \%$ (91/113) of women, whereas $87.5 \%(28 / 32)$ of the women who delivered by cesarean section had an OSA $<121^{\circ}$.

Conclusions: Measurement of the OSA, by sonography is feasible, reproducible and an objective tool to assess the degree of fetal head deflexion during labour and to predict the mode of delivery.
\end{abstract}

Keywords: Dystocia, Fetal attitude, First stage of labour, Labour arrest, Reproducibility, Transabdominal ultrasound

\section{INTRODUCTION}

With the widespread availability of ultrasonography devices, there has been an unprecedented increase in the importance of sonographic assessment as a valuable clinical tool, particularly in obstetric practice, due to its safety for use in pregnant women unlike other diagnostic modalities. Sonographic assessment of patients during early stages of labour has attracted considerable research interest, particular for the prediction of the progress and outcome of delivery. In fact, ultrasound evaluation during the first stage of labour has been found to be superior to vaginal examination in the detection of malpositions and in the subsequent prediction of the mode of delivery. ${ }^{1}$ 
Various parameters such as angle of progression, progression distance, head-symphysis distance has been found to of value in the prediction of the outcome of delivery. ${ }^{2,3}$ However, these parameters are useful when measured in the second stage of labour. There is certainly a need for early predictors.

The occiput-spine angle (OSA) has been recently reported as a new parameter that can help determine the likelihood of operative delivery. ${ }^{4}$ The OSA is determined as the angle formed by the occiput and spine of the fetus, as measured by sonography in the sagittal plane. Studies have shown an association between the value of OSA and the need for intervention, including cesarean section or instrumentation. ${ }^{4,5}$ However, there still remains some ambiguity regarding the manner in which the OSA influences the progress and outcome of delivery.

In this study, we aimed to determine whether the measurement of the OSA, as determined by sonographic evaluation, enabled the prediction of the mode of delivery, i.e., whether or not vaginal delivery would occur, in nulliparous pregnant women. In addition, we sought to evaluate the degree of intra- and interobserver variability in the OSA measurement in order to assess the reliability of the measurement of this parameter and thereby assess its value in clinical application.

\section{METHODS}

\section{Study protocol}

We conducted a prospective study using a sample of convenience comprising women attending our labour clinic at a tertiary health care center between July 2016 and June 2017. The study protocol was approved by the institutional ethics committee. Written informed consent for participation in this study was obtained from all the subjects.

\section{Inclusion criteria}

- Nulliparous women, uncomplicated, singleton pregnancies with cephalic presentation

- Gestational age of 37 to 41 weeks, as determined by the expected date of delivery calculated using dates of regular cycles

- Known last menstrual period (LMP), and first trimester ultrasound

- Spontaneous or induced labour pain (with 2 to 3 contractions every 10 minutes) with early active phase of labour (4-6 cm of cervical dilatation)

- Fetal head stationed above level 0, with or without membrane rupture, as assessed by vaginal examination

- The position of the fetal head was determined on the basis of the findings of transabdominal ultrasonography

\section{Exclusion criteria}

- Fetus was in the occiput-posterior position, because of the technical difficulties in determining the OSA in such cases

- Multiparous women

- Women with preterm labour, with established active phase

- Membrane rupture having occurred >24 hours prior

- Presence of abnormal fetal heart rate. Besides taking the patients' detailed medical history, we also performed initial vaginal examination to determine the status of the cervix (dilation, effacement, position of the external os) and position of the fetal head.

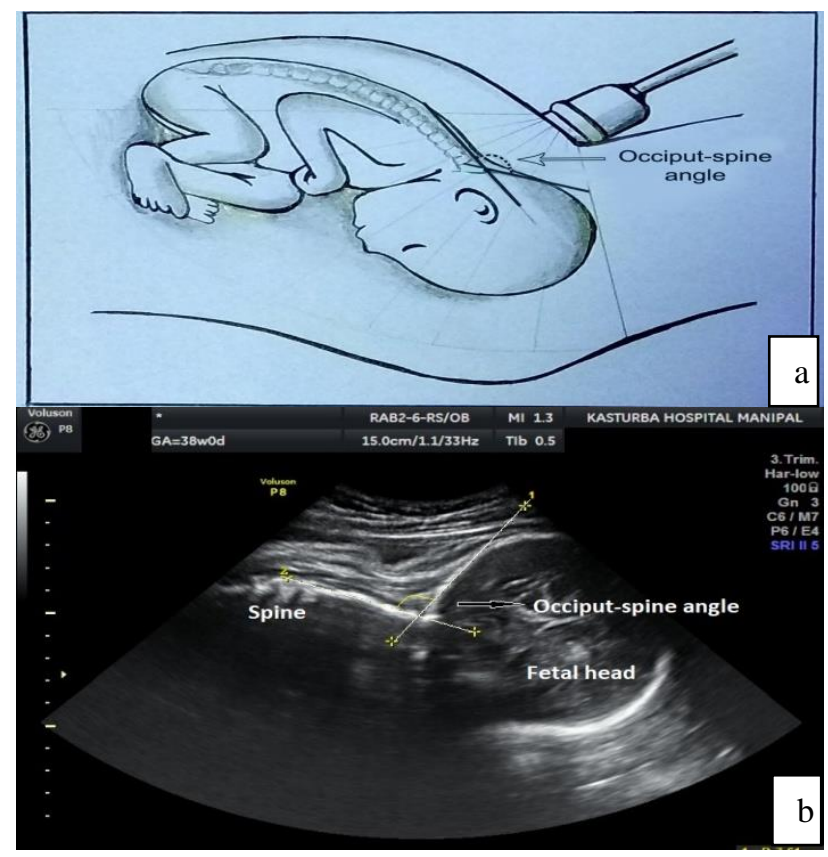

Figure 1: a) Schematic representation and b) Transabdominal ultrasound image of measurement of Occiput-spine angle.

\section{OSA measurement}

All patients underwent two-dimensional ultrasonography to acquire images showing the fetal head and upper spine. A portable ultrasonography machine, Voluson P8 series ultrasound systems equipped with a 4-7 MHz transabdominal two-dimensional convex transducer was used for all the examinations. The measurements were made during the active phase of the first stage of labour. The images were analyzed to calculate the OSA, which is the angle formed between the intersection of two tangential lines: one line across the fetal occipital bone and the other across the body of the first vertebra of the fetus (Figure $1 \mathrm{a}$ and Figure 1b). The OSA measurement was made in duplicate by two independent investigators to evaluate the intra- and interobserver agreement of the measurement. No changes were effected in the routine management of the labour on the basis of the results of the ultrasound measurement. The investigators were not 
involved in the clinical management of the patients, while the obstetrician treating the patients was blinded to the OSA measurement and findings of ultrasonography.

\section{Clinical assessment}

The progress of labour, labour outcome and mode of delivery for each patient were retrospectively evaluated by using partogram and standard proforma. Women who underwent delivery by cesarean section due to fetal distress were excluded from further assessment. Only cases in which cesarean delivery was performed due to labour dystocia were included in the present study because the study was focused on evaluating the correlation between the OSA and the mode of delivery.

\section{Cases of labour dystocia were those with any of the following}

- $\quad$ Prolonged first stage of labour (cervical dilation $<1.2$ $\mathrm{cm} /$ hour in nulliparous woman)

- Arrest of cervical dilation (cervical dilation remains the same even after more than 2 hours of good uterine contraction and rupture of membranes)

- Arrest of descent (lack of head descent in 2 hours in nulliparous woman despite good contractions and rupture of membranes)

- Prolonged second stage of labour ( $>2$ hours in nulliparous despite good uterine contraction and active pushing).

\section{Statistical analysis}

Intra and interobserver agreement in OSA measurement was assessed using Pearson's correlation coefficient (Pearson r). Interobserver agreement was assessed by plotting a Bland Altman graph and determining the limits of agreement, which represent the range within which lie $95 \%$ of the interobserver differences. The first measurement by expert operator was used for all subsequent analysis. The association between fetal OSA and fetal head station was evaluated using box plots. Comparisons between various parameters of patients who underwent vaginal delivery and those who underwent Cesarean delivery were made using the chi-square test, Fischer exact test, or Mann-Whitney U test, as appropriate.

The area under receiver operating characteristic (ROC) curve was determined to assess the value of OSA measurement in predicting whether vaginal delivery would occur or Cesarean delivery would be required. Comparison of the different values of the OSA according to mode of delivery in nulliparous pregnant women was made by using cross tabs and graphic representation using boxplots. A $\mathrm{P}$ value of $<0.05$ was considered statistically significant. The software package used for the statistical analysis was PASW statistics version 23.0 (SPSS Inc., Chicago, IL, USA).

\section{RESULTS}

Intra- and interobserver variability in OSA measurement: The occiput spine angle measurement yielded very good intraobserver agreement (Pearson=0.82 95\% CI 0.7280.88 ) and fair to good interobserver agreement (Pearson=0.62 95\% CI 0.51-0.71). The mean interobserver difference was $1.6^{\circ}$, and the limits of agreement were $-20^{\circ}$ to $17^{\circ}$ as described by Bland Altman (Figure 2).

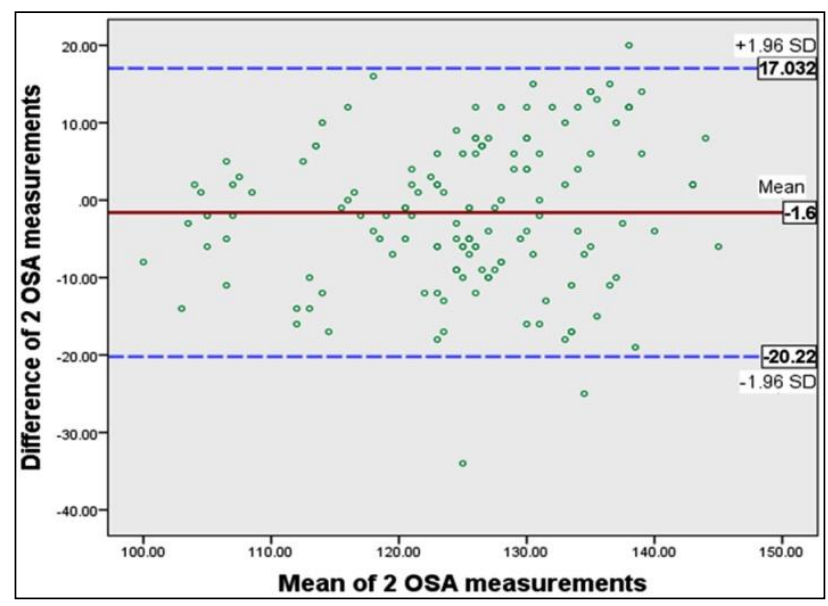

Figure 2: Bland Altmans graph showing limits of agreement between two observers (interobserver variation) for sonographic measurement of fetal occiput-spine angle.

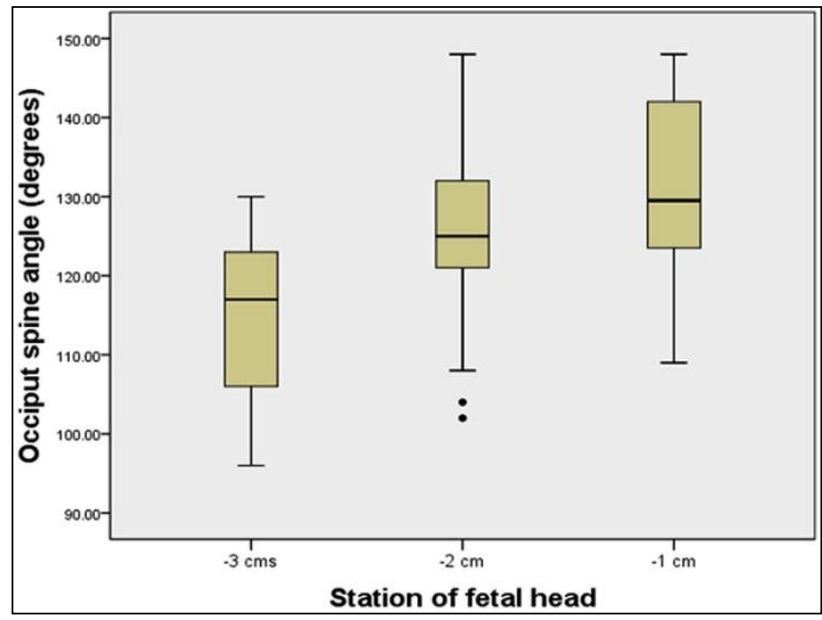

Figure 3: A box plot showing the distribution of occiput spine angle values according to the fetal head station.

\section{OSA measurement}

Follow up of all the 145 nulliparous pregnant women included in this study showed that 113 women had vaginal deliveries, whereas the remaining 32 required conversion to caesarean section due to labour arrest. Patients who underwent vaginal delivery and those who required cesarean section were compared for different 
demographic and clinical parameters. No difference was noted between the two groups in terms of the basic demographic characteristics such as age, BMI, and gestational age, thereby indicating that the two groups were comparable (Table 1).

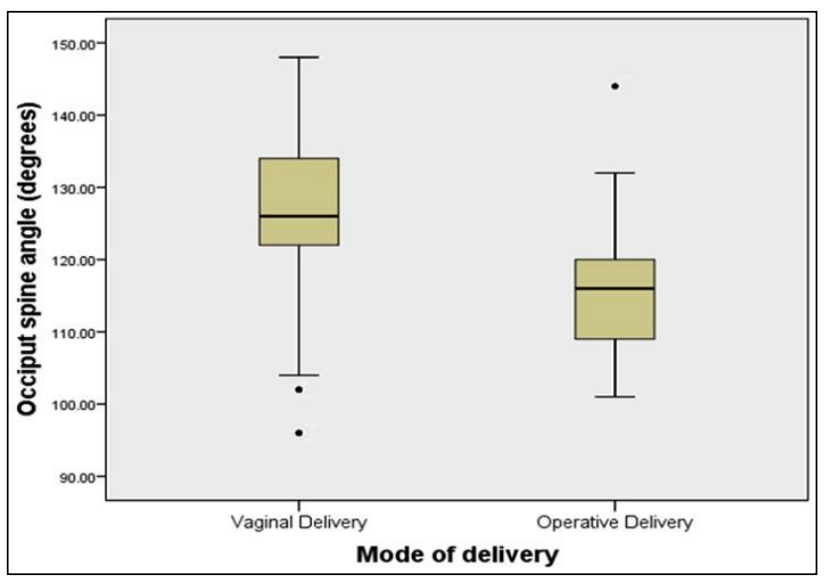

Figure 4: Occiput spine angle (OSA) on transperineal ultrasound in nulliparous women before onset of labour, comparing those who went on to deliver by cesarean section $(n=32)$ with those who delivered vaginally $(n=113)$.
However, due to labour arrest, the cesarean section group showed significant differences from the group who had vaginal delivery, in terms of the following variables: lower APGAR score at 5 minutes $(7.9 \pm 1.2$ versus $8.8 \pm 0.4)$ greater duration of both first stage $(431.03 \pm 78.4$ versus $312.83 \pm 74)$ and second stage of labour $(48.47 \pm 20.21$ versus $39.38 \pm 7.81)$.

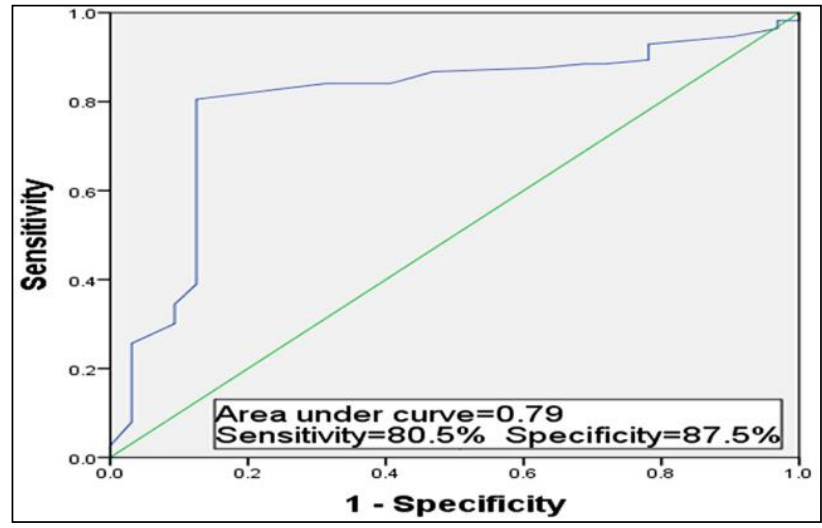

Figure 5: ROC for occiput spine angle in identifying the women who underwent cesarean section due to labour arrest (criterion: $\geq 121^{\circ}$, sensitivity $=\mathbf{8 0 . 5} \%$, specificity $=87.5 \%$ ).

Table 1: Maternal and obstetric characteristics stratified by type of delivery among nulliparous women, after excluding cesarean section done due to fetal distress cases.

\begin{tabular}{|c|c|c|c|c|}
\hline \multirow{2}{*}{ Characteristics } & All & Vaginal Delivery & Cesarean delivery & \multirow{2}{*}{$\mathbf{P} *$} \\
\hline & $(n=145)$ & $(\mathbf{n}=\mathbf{1 1 3})$ & $(n=32)$ & \\
\hline Age (years) & $26.2 \pm 3.3$ & $26.035 \pm 0.2$ & $27.31 \pm 0.7$ & 0.1 \\
\hline $\mathrm{BMI}\left(\mathrm{kg} / \mathrm{m}^{2}\right)$ & $24.20 \pm 3.6$ & $24.3 \pm 3.5$ & $23.6 \pm 4.0$ & 0.3 \\
\hline Gestational age at delivery(weeks) & $38.9 \pm 0.6$ & $38.8 \pm 0.9$ & $39.0 \pm 0.8$ & 0.4 \\
\hline \multicolumn{5}{|c|}{ Station of fetal head (centimeters above ischial spines) } \\
\hline $3 \mathrm{~cm}$ & 41 & $28 / 41(68.3 \%)$ & $13 / 41(31.7 \%)$ & \\
\hline $2 \mathrm{~cm}$ & 64 & $51 / 64(79.7 \%)$ & $13 / 64(20.3 \%)$ & \\
\hline $1 \mathrm{~cm}$ & 40 & $34 / 40(85 \%)$ & $6 / 40(15 \%)$ & 0.1 \\
\hline \multicolumn{5}{|l|}{ Onset of labour } \\
\hline Spontaneous & 105 & $81 / 105(77.1 \%)$ & $24 / 105(22.8 \%)$ & 0.7 \\
\hline Induction & 40 & $32 / 40(80 \%)$ & $8 / 40(20 \%)$ & \\
\hline \multicolumn{5}{|l|}{ Prelabour rupture of membrane } \\
\hline Present & 41 & & $10 / 41(24.4 \%)$ & 0.5 \\
\hline \multirow[t]{2}{*}{ Absent } & 104 & $31 / 41(75.6 \%)$ & $22 / 104(21.2 \%)$ & \\
\hline & & $83 / 104(79.8 \%)$ & & \\
\hline Occiput spine angle (OSA) & $124.2 \pm 11.5^{0}$ & $126.53 \pm 11.1^{0}$ & $116.25 \pm 9.2^{0}$ & $<0.01$ \\
\hline \multicolumn{5}{|l|}{ Duration of labour (mins) } \\
\hline 1st stage & $339.03 \pm 89.5$ & $312.83 \pm 74$ & $431.03 \pm 78.4$ & $<0.01$ \\
\hline 2nd stage & $41.37 \pm 12.21$ & $39.38 \pm 7.81$ & $48.47 \pm 20.21$ & 0.01 \\
\hline Birth weight(grams) & $2995 \pm 420$ & $2989 \pm 406$ & $3113 \pm 407$ & 0.1 \\
\hline APGAR score (@ 5mins) & $8.6 \pm 0.7$ & $8.8 \pm 0.4$ & $7.9 \pm 1.2$ & $<0.001$ \\
\hline
\end{tabular}

Data are presented as mean \pm SD or $\%$ or number, *Nulliparous women: vaginal vs. Cesarean delivery. BMI, body mass index; GA, gestational age; US, ultrasound 
A moderately positive correlation was noted between the OSA value and the fetal head station during active phase of first stage of labour, as assessed by digital examination (Pearson's correlation coefficient $0.54, \mathrm{P}=<0.01$ ). More specifically, the lower the fetal station at the time of ultrasound assessment, the wider was the OSA value (Figure 3). Interestingly, there was no increase in the risk of cesarean section in cases of high fetal station which was determined by digital examination.

The frequency of the different occiput positions were as follows: anterior $(9.6 \%)$, right-transverse $(15.8 \%)$, right anterior $(11.03 \%)$, left transverse $(33.79 \%)$, and left anterior $(29.65 \%)$. The OSA measurements for the study group for different types of fetal occiput positions were comparable. No significant intergroup differences were noted in the OSA measurements for the different occiput positions (analysis of variance $\mathrm{F}$-test $=0.319, \mathrm{P}=0.8$ ).

\section{Relationship between mode of delivery and OSA measurement}

A comparison between women who underwent cesarean section due to labour arrest and those who underwent vaginal delivery among nulliparous women is shown in Table 1 . The mean value of the OSA for the entire study population of nulliparous pregnant women was $124.2 \pm 11.5^{\circ}$. The mean OSA in the group of women with vaginal delivery was $126.53 \pm 11.1^{\circ}$, which was significantly greater than that in the group of women who had cesarean delivery $\left(116.25 \pm 9.2^{\circ}\right) ; \mathrm{P}<0.01$, as shown in Figure 4.

The area under the ROC curve for the prediction of vaginal delivery was $0.798(\mathrm{P}<0.001)$, as shown in Figure 5. A cut-off value of $121^{\circ}$ derived from the ROC curve was tested for its ability to differentiate between vaginal delivery and cesarean delivery in nulliparous women. This value had a sensitivity of $80.5 \%$, a specificity of $87.5 \%$, a positive predictive value of $94.7 \%$, and a negative predictive value of $54.53 \%$ in predicting vaginal delivery in full-term nulliparous women.

\section{DISCUSSION}

Various ultrasonographic parameters including angle of progression, progression distance, and head-symphysis distance have been reported to be useful in predicting the duration and progress of labour as well as in the evaluation of the risk of instrumentation or operative delivery. ${ }^{2,3}$ However, these parameters have been found to be useful when measured in the second stage of labour. ${ }^{3}$ Studies have shown than the determination of the occiput and spine position alone may not be useful in predicting the mode of delivery in nulliparas. ${ }^{6}$

Under these circumstances, the identification of a parameter that would enable such a prediction would be certainly desirable to allow obstetricians to sufficiently plan for operative management if the need arises. Thus, the OSA, measured in the active phase of the first stage of delivery, if found to be reliable and reproducible could be a very useful clinical tool for the prediction of the outcome of delivery.

In this study, we investigated whether OSA measurement during the active stage of the first stage of labour could help in the prediction of the mode of delivery. Analysis of area under ROC revealed a cut-off of $121^{\circ}$ for OSA in this study. This implies that an OSA of $<121^{\circ}$ was associated with Cesarean delivery in nulliparas. This cutoff is similar, but slight lower, that that reported by Maged et al, who reported a cut-off value of $126^{\circ} .^{5}$ Ghi et al reported a cut-off of $125^{\circ}$, which is again greater than the value obtained in our study. ${ }^{4}$ The differences between our study and those of Maged et al and Ghi et al may be attributed to the differences in the study populations of the studies., ${ }^{4,5}$ Both studies were inclusive of both multiparas as well as nulliparas, whereas ours was restricted to nulliparas since they were the focus group of the current study.

The sensitivity and specificity of the cut-off of $126^{\circ}$ reported by Maged et al was $78.4 \%$ and 93.79, respectively, while that of ours for a cut-off of $121^{\circ}$ was $80.5 \%$ and $87.5 \%$, implying that our cut-off had greater sensitivity, while theirs had better specificity. ${ }^{5}$ Therefore, accounting for the risk of Cesarean delivery in nulliparas if the OSA is between $121^{\circ}$ and $126^{\circ}$ could be considered as a reasonable guideline for clinicians.

To test for the reliability of the OSA measurement, we evaluated the inter- and intraobserver agreement. We found very good intraobserver agreement and fair to good interobserver in OSA measurement, as reported in previous studies. $^{4}$

With respect to the OSA measurements for different fetal head positions, we did not find any differences between women who had vaginal delivery and those who required Cesarean section in this study. However, one point to be taken into consideration in this regard is that we did not include patients with the occiput posterior position, and we cannot comment on whether there would be any difference in our findings if cases with this fetal position were also included.

In terms of fetal head station, we found that the lower the fetal head station, the greater was the OSA, which meant an increased possibility of vaginal delivery; in fact, a significant association was noted between the fetal head station and the OSA. This finding is consistent with those of previous studies, which indicate a risk of cesarean delivery with higher fetal station in nulliparas. ${ }^{6}$ Interestingly, the converse was not found to be true in our study, i.e., a higher fetal head station, as assessed by digital examination was not associated with increased risk of cesarean delivery. Therefore, it may be inferred that while a lower fetal head station is favorable to 
vaginal delivery, correlation with OSA would be more useful in assessing the risk of cesarean delivery.

Some of the limitations of our study are the small sample size and single-centre nature of the study. Further investigations conducted across multiple centers and including a greater number of subjects are warranted. Another limitation of this study is that we included only nulliparous women. However, this was because this study was focused on determined the cut-off value of OSA for this specific group of patients. Further, we excluded cases in which the fetus was in the occiput-posterior position. Therefore, there may be some degree of selection bias in this study. However, the measurement of the OSA is technically difficult in these cases, and even if they were included, and a suitable value was obtained, it would be practically difficult to apply the findings in actual clinical practice.

\section{CONCLUSION}

Our findings indicate that for nulliparous women, if the fetal occiput-spine angle measured by transabdominal ultrasound during the active phase of the first stage of labour is $<121^{\circ}$, there is an increased risk of cesarean delivery due to labour arrest. Our findings also confirm that the OSA measurement shows good intra- and interobserver reliability. Further, more large-scale studies are necessary to confirm our findings in order to ensure widespread applicability of our findings.

Funding: No funding sources Conflict of interest: None declared

Ethical approval: The study was approved by the Institutional Ethics Committee

\section{REFERENCES}

1. Malvasi A, Giacci F, Gustapane S, Sparic R, Barbera A. Intrapartum sonographic signs: new diagnostic tools in malposition and malrotation. J Matern Fetal Neonatal Med. 2016;29:2408-13.

2. Eggeb $\emptyset$ TM, Hassan WA, Salvesen KA, Lindtjørn E, Lees CC. Sonographic prediction of vaginal delivery in prolonged labour: a two-center study. Ultrasound Obstet Gynecol. 2014;43:195-201.

3. Ghi T, Maroni E, Youssef A, Morselli-Labate AM, Paccapelo A. Sonographic pattern of fetal head descent: relationship with duration of active second stage of labour and occiput position at delivery. Ultrasound Obstet Gynecol. 2014;44:82-9.

4. Ghi T, Bellussi F, Azzarone C, Krsmanovic J, Franchi L. The "occiput-spine angle": a new sonographic index of fetal head deflexion during the first stage of labour. Am J Obstet Gynecol. 2016;215:84.e1-7.

5. Maged AM, Soliman EM, Abdellatif AA, Nabil M, Said OI. Measurement of the fetal occiput-spine angle during the first stage of labour as predictor of the progress and outcome of labour. J Matern Fetal Neonatal Med. 2018;32:2332-7.

6. Kamel R, Youssef A. How reliable is fetal occiput and spine position assessment prior to induction of labour? Ultrasound Obstet Gynecol. 2018;53:53540.

Cite this article as: Somu K, Sujatha BS, Hebbar S, Shyamala G, Pai MV. Sonographic assessment of fetal head deflexion using occiput: spine angle measured during first stage of labour and its role in predicting the mode of delivery among nulliparous women. Int J Reprod Contracept Obstet Gynecol 2019;8:3025-30. 